\title{
Malignant melanoma mimic fungal infection a case report
}

\author{
Juan Sun ${ }^{1}$, Yun-Zhu Zhu' ${ }^{2}$ Pan-Pan Shao ${ }^{3}$, Jing Ke ${ }^{4}$, Wei Wang ${ }^{2}$, Qiu-Lin Sun ${ }^{2}$, Jia-Bin Li ${ }^{2}$ and Jun Cheng ${ }^{2 *}$
}

\begin{abstract}
Background: Most of malignant melanomas originate from skin and often metastasize to the lungs, rarely metastasizes to the liver and bone. However, imageology characters of lung metastasis tumor are commonly similar to those of fungal infections.

Case presentation: A patient was admitted with unhealed plantar puncture wound for 3 years, and cough and expectoration for 2 years. The chest computed tomography (CT) revealed multiple nodules with cavities, and the patient was diagnosed of pulmonary fungal infection in another hospital and received antifungal therapy for more than 8 months, but the clinical symptoms and chest imaging findings continue to progress. After admission, the pathological results of both lung biopsy and biopsy of the plantar wound 3 years ago indicated malignant melanoma.
\end{abstract}

Conclusions: The diagnosis of lung lesions cannot rely solely on imaging diagnosis, lung biopsy should be performed if necessary.

Keywords: Melanoma, Pulmonary fungal infection, Biopsy, Misdiagnosis

\section{Introduction}

In recent years, the morbidity and mortality of melanomas showed increasing trend, and for most patients, metastasis had already occurred before diagnosis was confirmed. Lung is one of the most common site of melanoma metastases. Imageology, such as chest computed tomography (CT), is one of the most commonly used examination methods to confirm the diagnosis of lung diseases [1-4]. However, imageology characters of pulmonary melanomas are similar to those of pulmonary infection, especially fungal infection, both of which showing nodules or cavity-like changes [5-8]. Accordingly, it is often required to combine blood test results and pathological findings to make a clear diagnosis. Here, we presented a case of a middle-aged man with recurrent cough and expectoration, after receiving anti-

\footnotetext{
* Correspondence: chengjun611@sina.com

${ }^{2}$ Department of Infectious Diseases, the First Affiliated Hospital of Anhui Medical University, Anhui Province 230022 Hefei, P. R. China

Full list of author information is available at the end of the article
}

fungal therapy for more than 8 months at a local hospital, finally diagnosed as melanoma with multiple metastases.

\section{Case presentation}

On January 8th, 2021, a 58-year-old male patient was admitted to the Department of Infectious Diseases, the First Affiliated Hospital of Anhui Medical University due to recurrent cough and expectoration for 2 years and a significant deterioration in corresponding symptoms a month ago.

Medical history showed that on March 8th, 2020, at a local hospital, his chest CT revealed multiple abnormal density shadows in both lungs, indicating possible fungal infection. His left heel was punctured by an iron nail on a mushroom farm 3 years ago (Fig. 1). The wound remained unhealed with recurrent ulcer. Wound secretions from skin lesions were cultured and Candida albicans was identified. Gram-positive cocci and fungal spores were found using sputum smear microscopy. 


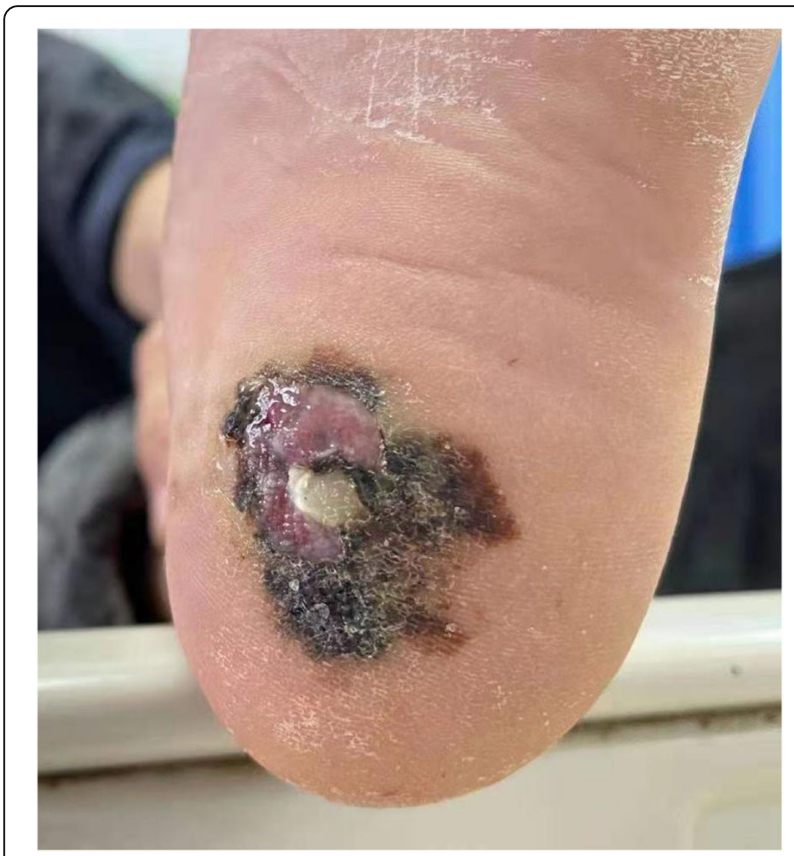

Fig. 1 Plantar wound

Fungal infection of the left heel accompanied by intrapulmonary dissemination was considered and fluconazole anti-fungal treatment was performed. Six months later, his symptoms of cough and expectoration were not significantly relieved, and chest CT result was worse than before (Fig. 2). And then, the anti-fungal agent was changed into voriconazole. The patient experienced pain in the right lower abdomen with obvious tenderness during coughing on December 25th, 2020. He went to the local hospital for further check. Multiple infectious lesions in the liver, liver abscess from some lesions, splenomegaly, and low density shadow of spleen were found using abdominal ultrasonography, indicating that there may be infections.

Accordingly, he was referred to our hospital. On admission, except for low fever, his vital signs were basically normal with body temperature at $37.8{ }^{\circ} \mathrm{C}$, blood pressure at $109 / 84 \mathrm{mmHg}$, heart rate at $101 \mathrm{beat} / \mathrm{min}$, and respiration rate at $23 \mathrm{time} / \mathrm{min}$. No obvious abnormalities in lungs, soft abdomen, tenderness in the right lower abdomen, positive percussion pain in the liver area, and palpable spleen under the ribs were found through physical examination. An ulceration with an area of $4 \mathrm{~cm} \times 5 \mathrm{~cm}$ was observed on his left heel. There were dark necrosis area surrounding the wound and bloody pus discharged from the center. Blood routine was basically normal with white blood cell counts of $7.10 \times 10^{9} / \mathrm{L}$, hemoglobin of $128.00 \mathrm{~g} / \mathrm{L}$, and platelet counts of $1.71 \times 10^{11} / \mathrm{L}$. Lower albumin level $(31.80 \mathrm{~g} / \mathrm{L})$ and no significant abnormalities in liver and kidney functions were found. For inflammatory indicators, higher C-reactive protein (CRP) level (43.77 mg/L), higher erythrocyte sedimentation rate (ESR) level $(56.00 \mathrm{~mm} / \mathrm{h})$, and no obvious increase in procalcitonin level $(0.18 \mathrm{ng} / \mathrm{mL})$ were observed. Cryptococcus capsular antigen test, $\mathrm{G}$ test, and GM test were all negative. Gram-positive cocci and gram-negative bacilli were found by sputum smear microscopy, while mycete and tubercle bacillus were not detected. Blood cultures were negative. For the tumor indicators, neuron-specific enolase $(60.65 \mathrm{ng} / \mathrm{mL})$ and ferritin $(437.80 \mathrm{ng} / \mathrm{mL})$ increased.

He was preliminarily diagnosed as pulmonary fungal infection and suspected liver abscess after admission. Voriconazole was used for anti-infective treatment. Because he was unresponsive to anti-fungal therapy for 8 months, malignancy could not be ruled out. Lung and wound tissue biopsies were performed, and samples were sent for PACEseq metagenomic next-generation sequencing (mNGS) analysis (Hugobiotech, Beijing, China). Meanwhile, magnetic resonance imaging (MRI) showed multiple abnormal signals in both lungs, liver, thoracic and lumbar vertebrae, and multiple small and medium-sized lymph nodes in the retroperitoneum (Fig. 2). Chest CT plain scan plus enhanced scan indicated multiple nodules and clumpy high-density shadows in both lungs with blurred boundaries, and cloudy fuzzy shadows in the surrounding areas (Fig. 2). After enhancement, mild consolidation was observed and multiple cavities were visible inside. The above results of imageology indicated possible fungal infection. However, no pathogens were detected in lung biopsy tissues by mNGS. Accordingly, both pathological (tissue biopsy of both lungs and unhealed plantar puncture wound) and immunohistochemical results supported the diagnosis of malignant melanoma (Fig. 3). The liver and thoracolumbar lesions might be considered as results of melanoma metastases. However, he rejected our recommendation to perform liver biopsy. With consultation to the oncology department, it was concluded that his melanoma was accompanied by systemic metastases and immunotherapy might be feasible. However, considering the poor prognosis and the high cost, the patient and his family abandoned treatments and requested discharge after thorough considerations.

\section{Discussion}

Melanomas originate from neural crest melanocytes, and are usually derived from benign growth-stunted mole. The pathogenesis of melanomas is associated with genetic and environmental factors, among which family history is the biggest risk factor. Other factors include 


\section{a}

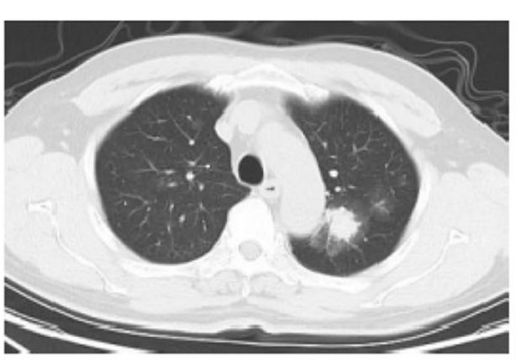

c

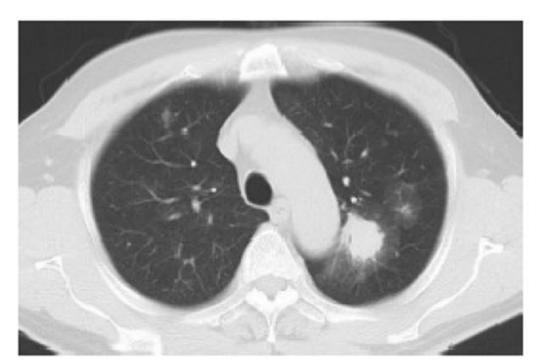

e

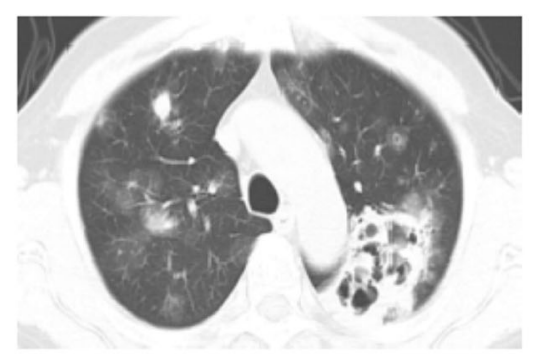

g

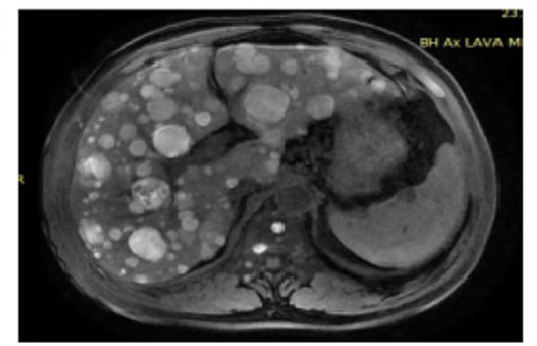

b

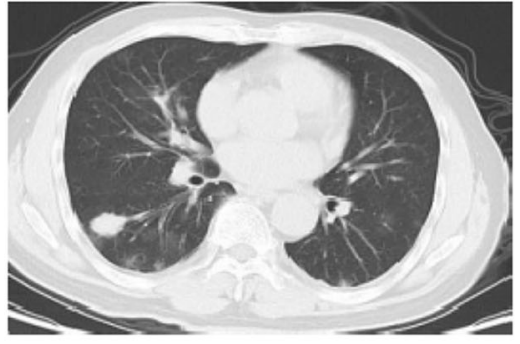

d

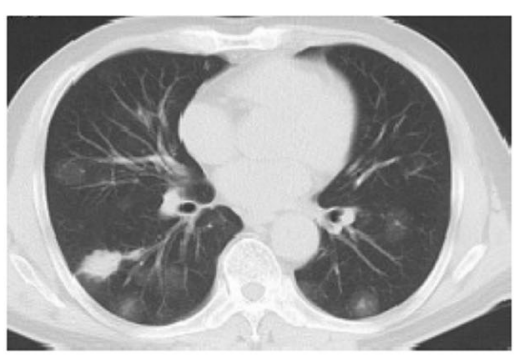

f

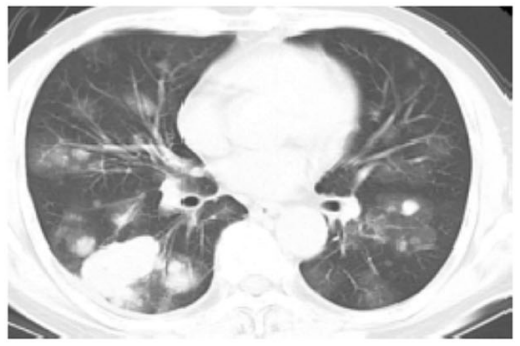

h

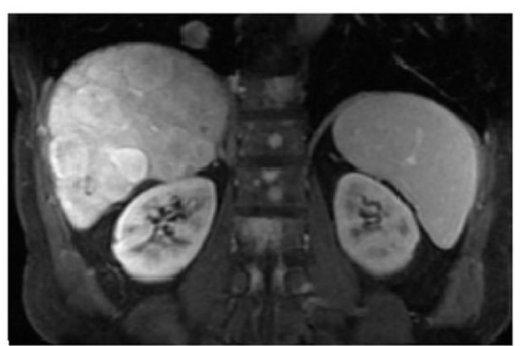

Fig. 2 Relevant imageology characters during the patient's visit. A-B, Chest CT on March 16th, 2020. C-D, Chest CT on June 2nd, 2020. E-F, Chest CT on Janurary 12th, 2021. G-H, Upper abdominal enhanced MRI on Janurary 10th, 2021

ultraviolet radiation, skin photosensitivity, autoimmune state, and the presence of melanocytes or dysplastic moles $[2,4,9,10]$. The pathogenesis of malignant melanomas is very complex, including a series of complicated interactions among external events and endogenous triggers, genetic mutations of BRAF, NRAS, and KIT, as well as endogenous and immune-related factors of the tumor $[2,10]$. Approximately $90 \%$ of malignant melanomas originate from the skin, while melanomas of lung and liver origin are very rare $[3,11,12]$. In this report, tissue biopsy results of both lungs and unhealed plantar puncture wound indicate the patient should be diagnosed as malignant melanoma.

This patient had a plantar puncture wound 3 years ago and manifested pulmonary symptoms such as coughing and expectoration about 1 year later. However, without enough attention and proper care, concomitant infection lead to persistently unhealed wound. The wound was at the heel, which was repeatedly compressed and rubbed during daily activities. Under the combined actions of multiple factors, normal cells mutated into tumor cells, which gradually invaded into the dermis. Subsequently, 


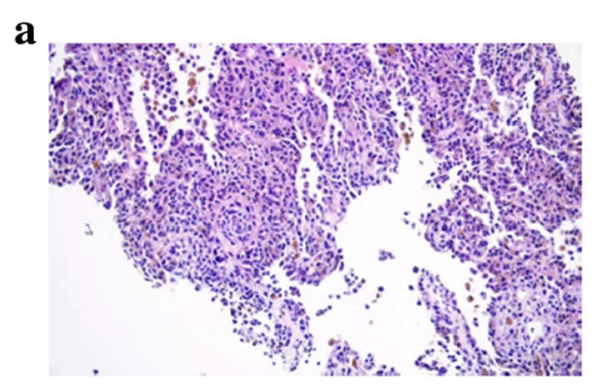

c

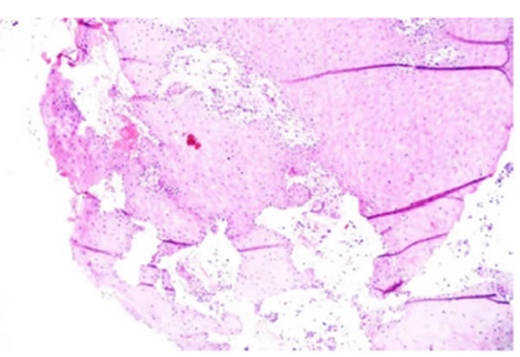

b

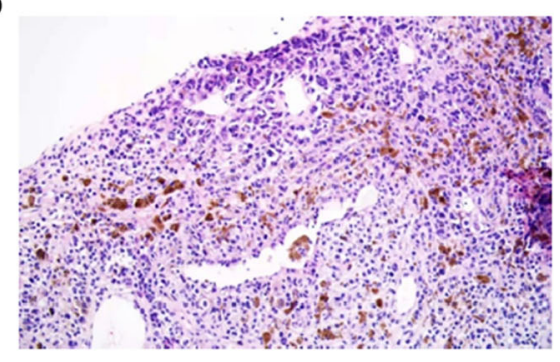

d

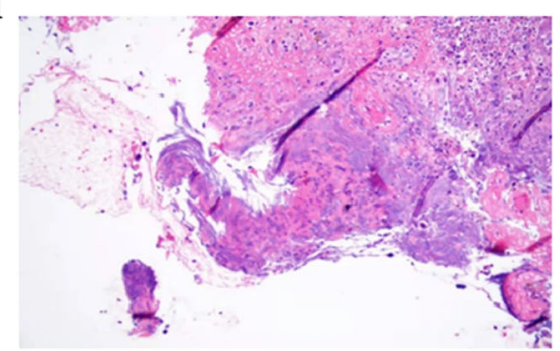

Fig. 3 Pathological images. A and $\mathbf{B}$ were pathological images of lung biopsy. C and $\mathbf{D}$ were pathological images of plantar wound biopsy

unhealed wound can help the tumor cells get in touch with blood and lymphatic systems and gradually spread from the original site [9]. In this case, there were no mole cells in the plantar lesion, and the change of normal cells into tumor cells may be stimulated by the protracted wound, which was different from the published pathogenesis of melanomas [2].

Due to the highly invasive and metastatic characteristics of malignant melanoma, for most of patients, metastasis has already occurred before diagnosis is confirmed. The lung and pleura are the most common sites of melanoma metastases and usually the primary sites of initial metastasis, possibly due to the convergence of blood and lymphatic systems, and the peripheral blood pumped from the right heart through the pulmonary artery $[4,9]$. Clinically, about $10-20 \%$ of the patients with malignant melanomas have concurrent liver metastasis [13], while $5-17 \%$ of the patients with stage IV melanomas have bone metastasis [14], which usually occur in the spine, pelvis, shoulder, and distal femur [15]. In this case, the patient has developed lung, liver, and thoracolumbar lesions, consistent with the published results.

For the patient in this case, it took nearly one year from the first visit due to cough and expectoration to the final diagnosis.The lung biopsy was not performed by the local doctor to confirm the diagnosis. Pathological diagnosis is the "gold standard" for pulmonary spaceoccupying lesions. For pulmonary fungal infection, scattered nodules, halo sign or crescent sign can be detected using CT scan $[7,8,16,17]$. According to the diagnostic criteria specified by the European Fungal Research Organization for Cancer Therapy (EORTC), the crescent sign and the presence of cavities in pulmonary lesions are typical manifestations of pulmonary fungal infection [16].

In this case, the above characters were all detected using CT scan. Wound secretions from skin lesions were cultured by the local hospital and C. albicans was identified. Accordingly, it was considered as pulmonary fungal infection through skin lesions into the blood. However, lung biopsy was not performed at that time to confirm the diagnosis. For lung metastases, the most common manifestations are multiple pulmonary nodules, while pulmonary cavities may also occur $[6,7,18]$. Imageology characters of lung metastases are similar to those of pulmonary infection. Thus, it is quite difficult to make an accurate diagnosis only by imageology. The state-of-theart mNGS technology would identify etiological pathogens, while the negative results might also provide useful information. In this case, the negative result of the lung biopsy further confirmed our diagnosis that the lung lesions were not caused by infection. In summary, before making a final diagnosis, the doctor should comprehensively evaluate clinical manifestations and examine the patient, especially the pathological results.

\section{Supplementary information}

The online version contains supplementary material available at https://doi. org/10.1186/s13000-022-01214-7.

\section{Additional file 1}

Acknowledgements

None. 


\section{Authors' contributions}

Prof. Jun Cheng contribute substance to ideas and design. Juan Sun draft the paper, Yun-Zhu Zhu, Pan-Pan Shao, Jing Ke, Wei Wang are responsible for collecting the clinical isolates, Qiu-Lin Sun and Jia-Bin Li give a lot of assistance and revise manuscript. The author(s) read and approved the final manuscript.

\section{Funding}

None.

\section{Availability of data and materials}

All data are fully available without restriction.

\section{Declarations}

Ethics approval and consent to participate

All procedures performed in studies involving human participants were in accordance with the ethical standards of the institutional and/or national research committee and with the 1964 Helsinki declaration and its later amendments or comparable ethical standards. All subjects signed an informed consent form. This study was approved by the Ethics Committee of the first affiliated hospital of Anhui Medical University.

\section{Consent for publication}

All authors consent for publication.

\section{Competing interests}

None.

\section{Author details}

'Department of Science and Technology, Anhui University of Chinese Medicine, 230038, Anhui Province, Hefei, P. R. China. ${ }^{2}$ Department of Infectious Diseases, the First Affiliated Hospital of Anhui Medical University, Anhui Province 230022 Hefei, P. R. China. ${ }^{3}$ Department of Infectious Diseases, Bozhou Peoples Hospital, 236803 Bozhou, Anhui Province, P. R. China.

${ }^{4}$ Pathology Department, the First Affiliated Hospital of Anhui Medical University, 230022, Anhui Province, Hefei, P. R. China.

Received: 24 August 2021 Accepted: 9 February 2022

Published online: 27 February 2022

\section{References}

1. Siegel RL, Miller KD, Jemal A. Cancer statistics. CA A Cancer J Clin. 2019;69: 7-34.

2. Schadendorf D, van Akkooi ACJ, Berking C, et al. Melanoma Lancet. 2018; 392:971-84.

3. Paliogiannis P, Fara AM, Pintus G, et al. Prim Melanoma Lung: Syst Rev Med (Kaunas). 2020;56:576.

4. Tas F. Metastatic behavior in melanoma: timing, pattern, survival, and influencing factors. J Oncol. 2012;2012:647684.

5. Georgiadou SP, Sipsas NV, Marom EM, et al. The diagnostic value of halo and reversed halo signs for invasive mold infections in compromised hosts. Clin Infect Dis. 2011:52:1144-55.

6. Shroff GS, Benveniste MF, Carter BW, et al. Imaging of Metastases in the Chest: Mechanisms of Spread and Potential Pitfalls. Semin Ultrasound CT MR. 2017;38:594-603

7. Gafoor K, Patel S, Girvin F, et al. Cavitary Lung Diseases: A Clinical-Radiologic Algorithmic Approach. Chest. 2018;153:1443-65.

8. Badiee P, Hashemizadeh Z. Opportunistic invasive fungal infections: diagnosis \& clinical management. Indian J Med Res. 2014;139:195-204

9. Damsky WE, Rosenbaum LE, Bosenberg M. Decoding melanoma metastasis. Cancers (Basel). 2010;3:126-63.

10. Lugović-Mihić L, Ćesić D, Vuković P, et al. Melanoma Development: Current Knowledge on Melanoma Pathogenesis. Acta Dermatovenerol Croat. 2019; 27:163-8.

11. Ao W, Wang J, Mao G, et al. Primary hepatic melanoma: A case report of computed tomography and magnetic resonance imaging findings. Med (Baltim). 2019;98:e16165.

12. Nakagawa T, Yasuda $Y$, Sugiyama $S$, et al. A case of malignant melanoma simultaneously found in the lung and small intestine. Clin J Gastroenterol. 2013;6:25-8
13. Leonardi GC, Falzone L, Salemi $R_{1 \prime}$ et al. Cutaneous melanoma: From pathogenesis to therapy (Review). Int J Oncol. 2018;52:1071-80.

14. Rustagi T, Yener U, Blakaj D, et al. Long-Term Survival After Brain and Spine Metastasis in Malignant Melanoma. World Neurosurg. 2019;125:164-9.

15. Migliorini F, Maffulli N, Trivellas A, et al. Bone metastases: a comprehensive review of the literature. Mol Biol Rep. 2020:47:6337-45.

16. Wang J, Zhang C, Lin J, et al. Clinical diagnostic value of spiral CT in invasive pulmonary fungal infection. Exp Ther Med. 2019;17:4149-53.

17. Zhan X, Zhang L, Wang Z, et al. Reversed halo sign: presents in different pulmonary diseases. PLOS ONE. 2015;10:e0128153.

18. Dwyer AJ, Reichert CM, Woltering EA, et al. Diffuse pulmonary metastasis in melanoma: radiographic-pathologic correlation. AJR Am J Roentgenol. 1984; 143:983-4.

\section{Publisher's Note}

Springer Nature remains neutral with regard to jurisdictional claims in published maps and institutional affiliations.
Ready to submit your research? Choose BMC and benefit from:

- fast, convenient online submission

- thorough peer review by experienced researchers in your field

- rapid publication on acceptance

- support for research data, including large and complex data types

- gold Open Access which fosters wider collaboration and increased citations

- maximum visibility for your research: over $100 \mathrm{M}$ website views per year

At BMC, research is always in progress.

Learn more biomedcentral.com/submissions 